\title{
Expression of galectin-3 in the tumor immune response in colon cancer
}

\author{
Patrick Dumont ${ }^{1}$, Alix Berton ${ }^{1}$, Nathalie Nagy ${ }^{1}$, Flavienne Sandras ${ }^{1}$, Sandrine Tinton ${ }^{1}$, Pieter Demetter ${ }^{1}$, \\ Françoise Mascart ${ }^{2}$, Abdelmounaaïm Allaoui ${ }^{3}$, Christine Decaestecker ${ }^{4}$ and Isabelle Salmon ${ }^{1}$
}

The role of tumor-associated macrophages (TAMs) is controversial. Although most studies on different cancer types associate them with a poorer prognosis, interestingly in colon cancer, most articles indicate that TAMs prevent tumor development; patients with high TAMs have better prognosis and survival rate. M1-polarized macrophages produce high level of tumor necrosis factor- $\alpha$, interleukin- $1 \beta$ or reactive oxygen species, which can effectively kill susceptible tumor cells. In contrast, M2-polarized macrophages can secrete different factors that promote tumor cell growth and survival or favor angiogenesis and tissue invasion. Considering the beneficial role of TAMs in colon cancer, we speculated that they may not display the M2 polarization commonly observed in tumor microenvironment, but rather develop M1 properties. Therefore, we used an in vitro model to analyze the effects of supernatants from M1-polarized macrophages on DLD-1 colon cancer cells. Our data indicate that the conditioned medium from LPS-activated macrophages (CM-LAM) contains a high level of granulocyte-macrophage colony-stimulating factor, interleukins $-1 \beta,-6,-8$ and tumor necrosis factor- $\alpha$, and that it exerts a marked growth inhibitory activity on DLD-1 cells. Prolonged exposure to CM-LAM results in cell death by apoptosis. Such exposure to CM-LAM leads to the modulation of gal-3 expression: we observed a marked downregulation of gal-3 mRNA and protein expression following CM-LAM treatment. We also describe that the knockdown of gal-3 sensitizes DLD-1 cells to CM-LAM. These data suggest an involvement of gal-3 in the response of colon cancer cells to proinflammatory stimuli, such as the conditioned medium from activated macrophages.

Laboratory Investigation (2008) 88, 896-906; doi:10.1038/labinvest.2008.54; published online 9 June 2008

KEYWORDS: galectin-3; immune response; colon cancer; macrophages; M1 polarization

Several lines of evidence indicate that immune effector cells play an important role in the recognition and destruction of cancer cells. This concept of cancer immunosurveillance is supported by human studies showing that immunodeficiencies predispose patients to the development of cancer and by murine models where inactivation of specific genes coding for components of the immune response results in a higher susceptibility to the development of spontaneous or chemically induced cancers. ${ }^{1,2}$ However, other studies are consistent with the view that immune responses may favor the progression of malignancies. ${ }^{3,4}$ Persistent immune responses exacerbate the recruitment and activation of immune cells in tumor microenvironments where they can contribute to the selection of nonimmunogenic tumor cell variants as well as to the supply of elements favoring tumor expansion. Indeed, many cancers arise at sites of infection or chronic inflammation.,

With regard to the role of macrophages, which usually constitute a significant part of the tumor-infiltrating immune cells, their presence has been associated with good prognosis for patients with colon cancers. ${ }^{7-10}$ This somehow constitutes an exception because it is the common view that tumorassociated macrophages (TAMs) promote tumor progression; the majority of studies on different cancer types including breast, prostate, bladder, cervical and kidney cancers have correlated high TAMs density with reduced patient survival. ${ }^{11,12}$ Classical morphological analyses of a tumor for the presence of TAMs do not, however, allow for distinction among the types of polarization of these macrophages. Macrophages can produce various agents such as reactive

\footnotetext{
'Laboratory of Pathology, Erasme Hospital, Université Libre de Bruxelles, Brussels, Belgium; ${ }^{2}$ Laboratory of Vaccinology and Mucosal Immunity, Erasme Hospital, Université Libre de Bruxelles, Brussels, Belgium; ${ }^{3}$ Laboratory of Molecular Bacteriology, Faculty of Medicine, Université Libre de Bruxelles, Brussels, Belgium and ${ }^{4}$ Laboratory of Toxicology, Institute of Pharmacy, Université Libre de Bruxelles, Brussels, Belgium

Correspondence: Professor I Salmon, MD, PhD, Laboratory of Pathology, Erasme Hospital, Université Libre de Bruxelles, 808 Lennik Road, Brussels 1070, Belgium. E-mail: Isabelle.Salmon@erasme.ulb.ac.be 
oxygen species, nitric oxide, tumor necrosis factor- $\alpha$ (TNF- $\alpha$ ) and interleukin- $1 \beta$ (IL-1 $\beta$ ) that can effectively kill cancer cells, when they have been activated with various stimuli, including lipopolysaccharide (LPS) or interferon- $\gamma$ (IFN- $\gamma$ ), that induce the M1 type of polarization. ${ }^{12-14}$ However, TAMs commonly adopt an alternative phenotype, the M2 polarization, as a consequence of factors present in the tumor microenvironment. ${ }^{12}$ This M2 polarization is linked to the ability of TAMs to release various factors such as plateletderived growth factor, fibroblast growth factor-basic, epidermal growth factor, vascular endothelial growth factor, IL-10, transforming growth factor- $\beta$ (TGF- $\beta$ ) and several matrix metalloproteases that can promote tumor cell growth and survival, angiogenesis, tissue invasion and suppression of the immune response. ${ }^{11,12}$

To study the role of infiltrating macrophages on the development of colon cancer, we chose to use a M1 type of model because TAMs appear to behave differentially in colon cancer than in other types of cancer. In this model, the human monocytic leukemia cell line THP-1 is induced to undergo differentiation into macrophages by treatment with phorbol ester, which has been reported to mimic native monocyte-derived macrophages. ${ }^{15}$ These macrophages subsequently activated with LPS secrete various cytokines such as IL- $1 \beta$ and TNF- $\alpha,{ }^{13,14}$ allowing the analysis of the effects of supernatants from M1-polarized macrophages on colon cancer cells.

Using this in vitro model, we characterized how the supernatants of M1-polarized macrophages modulate the expression and secretion of galectin-3 (gal-3) in colon cancer cells. We previously published that gal-3 is involved in colon cancer development and that the level of gal-3 expression in colorectal tumors is related to their level of aggressiveness. ${ }^{16-18}$ Other teams also showed that gal-3 is commonly expressed at a high level by colon cancer cells and that strong expression of gal-3 correlates with disease progression, metastasis and poor survival. ${ }^{19,20} \mathrm{Gal}-3$ is a member of the $\beta$-galactosidebinding lectin family and is expressed by many cell types including immune cells such as macrophages as well as many cancer cells. ${ }^{21}$ Due to its pleiotropic effects on processes, such as cell adhesion, induction of cytokine production, inflammation, chemotaxis, apoptosis, differentiation or regulation of gene expression, gal-3 is involved in many cellular functions that are crucial to cancer progression. ${ }^{21-25}$ We hypothesize that gal-3 plays a key role in the various crosstalks that exist between the infiltrating macrophages and the colon cancer cells, and in the regulation of the tumor immune response in colon cancer.

\section{MATERIALS AND METHODS Cell Culture}

THP-1 human monocytic leukemia cells (ATCC TIB-202) were maintained at $37^{\circ} \mathrm{C}$ in a $5 \% \mathrm{CO}_{2}$ humidified atmosphere, in RPMI 1640 medium supplemented with $10 \%$ fetal bovine serum (FBS), $0.05 \mathrm{mM}$ 2-mercaptoethanol, $100 \mathrm{U} / \mathrm{ml}$ penicillin G, $100 \mu \mathrm{g} / \mathrm{ml}$ streptomycin-sulfate and $250 \mathrm{ng} / \mathrm{ml}$ amphotericin B. The human colon adenocarcinoma cell line DLD-1 (ATCC CCL-221) was maintained in RPMI 1640 medium supplemented with $10 \% \mathrm{FBS}, 1 \mathrm{mM}$ sodium pyruvate, $10 \mathrm{mM}$ HEPES, $100 \mathrm{U} / \mathrm{ml}$ penicillin G, $100 \mu \mathrm{g} / \mathrm{ml}$ streptomycin-sulfate and $250 \mathrm{ng} / \mathrm{ml}$ amphotericin B. The cell culture media were obtained from Invitrogen.

\section{Differentiation of THP-1 Cells into Macrophages and LPS Activation}

THP-1 cells were differentiated into macrophages by treatment with $100 \mathrm{ng} / \mathrm{ml}$ phorbol-12-myristate-13 acetate (PMA; Sigma-Aldrich) for $48 \mathrm{~h}$. The PMA-containing medium was removed and replaced by complete medium. Macrophages were either maintained unstimulated or activated by treatment with $1 \mu \mathrm{g} / \mathrm{ml}$ LPS (Sigma-Aldrich). After $24 \mathrm{~h}$, the conditioned media of both unstimulated (CM-UM) and LPS-activated macrophages (CM-LAM) were collected, centrifuged at $10000 \mathrm{~g}$ for $10 \mathrm{~min}$ and stored at $-20^{\circ} \mathrm{C}$ until use. To assess the M1 polarization, the concentrations of IL-8, IL-6, granulocyte-macrophage colony-stimulating factor (GM-CSF), TNF- $\alpha$ and IL- $1 \beta$ were determined in the conditioned media by Bio-Plex Cytokine Immunoassays (Bio-Plex suspension array system, Luminex 100; Bio-Rad Laboratories, Nazareth-Eke, Belgium), using the antibodies from Invitrogen according to the manufacturer's instructions.

\section{MTT Assay}

Cells were seeded at 5000 cells per well in 96-well plates and submitted $24 \mathrm{~h}$ later to treatments with CM-UM and CM-LAM. At the end of the treatments, cells were incubated with complete RPMI medium containing $0.5 \mu \mathrm{g} / \mathrm{ml}$ (3-(4, 5-dimethylthiazolyl-2)-2,5 diphenyltetrazolium bromide (Sigma-Aldrich). After $3 \mathrm{~h}$, we discarded the MTT solution, solubilized the formazan produced by the living cells with a solution of isopropanol/1 $\mathrm{M} \mathrm{HCl}(24: 1 \mathrm{v} / \mathrm{v})$ and read the absorbance at $570 \mathrm{~nm}$ on a spectrophotometer (Genesis, $10 \mathrm{UV}$ ).

\section{Annexin V Binding Assay}

Cells were harvested by trypsinization, resuspended in FBSsupplemented medium to neutralize the trypsin and washed twice with phosphate buffered saline (PBS). Cells were resuspended in ice-cold $1 \times$ binding buffer (Sigma-Aldrich) at a density of $1 \times 10^{6}$ cells per ml. An aliquot of $500 \mu \mathrm{l}$ was stained for $10 \mathrm{~min}$ at room temperature with $5 \mu \mathrm{l}$ annexin $\mathrm{V}$ (Ann V)-fluorescein isothiocyanate and $10 \mu \mathrm{l}$ propidium iodide (PI; Sigma-Aldrich). The fluorescence was analyzed immediately on an Epics XL-MCL flow cytometer (Beckman Coulter) equipped with a $488 \mathrm{~nm}$ argon laser.

\section{Video Cellular Microscopy}

We seeded DLD-1 cells at 60000 cells per $25 \mathrm{~cm}^{2}$ flask in RPMI medium containing 10\% FBS. After $72 \mathrm{~h}$, cells were 
rinsed with PBS and incubated with one of the following medium: (1) RPMI 1640 containing $0.2 \%$ FBS; (2) $0.2 \%$ FBS RPMI 1640 supplemented with 20\% (v/v) CM-UM; (3) 0.2\% FBS RPMI 1640 supplemented with 20\% (v/v) CM-LAM. The flasks were placed under phase-contrast microscopes coupled to video cameras and filmed for $48 \mathrm{~h}$ (1 image every $4 \mathrm{~min}$ ) as previously described. ${ }^{26}$ On the recorded still images from time points $0,12,24,36$ and $48 \mathrm{~h}$, we counted the number of cells to quantify the effect of the treatments on cell growth.

\section{Gal-3 ELISA}

DLD-1 cells seeded at $10^{5}$ cells per well in 6-well plates were treated $24 \mathrm{~h}$ later with CM-UM and CM-LAM. At time points 24,48 and $72 \mathrm{~h}$, cell culture supernatants were collected and centrifuged at $4^{\circ} \mathrm{C}$ for $15 \mathrm{~min}$ at $1000 \mathrm{~g}$. Gal-3 levels were measured with the Human Galectin-3 ELISA kit from Bender MedSystems, according to the manufacturer's instructions.

\section{Western Blotting}

Cells were scraped and centrifuged at $500 \mathrm{~g}$ for $5 \mathrm{~min}$ at $4^{\circ} \mathrm{C}$. The cell pellets were washed twice with ice-cold PBS and resuspended in ice-cold radio-immunoprecipitation assay buffer (50 mM Tris-HCl, pH 7.6, $150 \mathrm{mM} \mathrm{NaCl}, 1 \% \mathrm{NP}-40$, $1 \%$ sodium deoxycholate, $0.1 \%$ sodium dodecyl sulfate (SDS), $1 \mathrm{mM} \mathrm{Na}_{3} \mathrm{VO}_{4}, 1 \mathrm{mM} \mathrm{NaF}$ ) supplemented with protease inhibitors (Protease Inhibitor Cocktail from Roche Diagnostics). The protein content of the lysates was measured by the Bradford method using the Dc Protein Assay kit (Bio-Rad Laboratories). We loaded $50 \mu \mathrm{g}$ of proteins on SDS-PAGE and the separated proteins were transferred onto Hybond C-extra nitrocellulose membranes (Amersham). Blots were blocked for $30 \mathrm{~min}$ in PBS containing $0.1 \%$ Tween-20 (PBS-T), 2\% nonfat dry milk and $2 \%$ bovine serum albumin (BSA). After blocking, the membranes were probed for $1 \mathrm{~h}$ with the primary antibody diluted in PBS-T containing $2 \%$ nonfat dry milk and $2 \%$ BSA. The membranes were washed three times for $20 \mathrm{~min}$ with PBS-T and probed with horseradish peroxidase-conjugated secondary antibody used at a 1:10 000 dilution in PBS-T containing 2\% nonfat dry milk and $2 \%$ BSA. After three washes in PBS-T, the membranes were developed using chemiluminescent substrate (Western Lighting from PerkinElmer) and exposed to Amersham ECL Hyperfilms. The following primary antibodies were used in this study: mouse anti-gal-3 antibody (dilution of 1:1000; Novocastra Laboratories), anti-Lamin A/C mouse ab (dilution of 1:400; Santa Cruz Biotechnology), anti-PARP (19F4) mouse ab (dilution of 1:2000; Cell Signalling) and anti- $\beta$-actin (AC-15) mouse ab (dilution of 1:5000; Sigma-Aldrich).

\section{Cell Fractionation}

Cells were scraped and centrifuged at $500 \mathrm{~g}$ for $10 \mathrm{~min}\left(4^{\circ} \mathrm{C}\right)$. Cell pellets were washed twice with ice-cold PBS and resuspended in 10 volumes of hypotonic buffer $(10 \mathrm{mM}$ HEPES, pH 7.9, $10 \mathrm{mM} \mathrm{KCl,} 0.1 \mathrm{mM}$ EDTA, $1 \mathrm{mM}$ DTT) supplemented with protease inhibitors. After allowing the cells to swell on ice for $15 \mathrm{~min}$, the plasma membranes were disrupted by repeated passages through a microtip. The samples were centrifuged at $4000 \mathrm{~g}$ for $2 \mathrm{~min}\left(4^{\circ} \mathrm{C}\right)$. The supernatants (cytoplasmic fraction) were collected and stored. The pellets were resuspended in ice-cold hypertonic buffer (20 mM HEPES, pH 7.9, 400 mM NaCl, 1 mM EDTA, 1 mM DTT, 20\% glycerol) supplemented with protease inhibitors. After incubation on ice for $30 \mathrm{~min}$ with frequent vortexing, the samples were centrifuged at $14000 \mathrm{~g}$ for $5 \mathrm{~min}\left(4^{\circ} \mathrm{C}\right)$. The supernatants (nuclear fraction) were isolated and stored.

\section{Total RNA Purification, cDNA Synthesis and Quantitative Real-Time PCR}

Total RNA was extracted with Trizol reagent (Invitrogen) according to the manufacturer's instructions, digested with DNAse I and purified on columns using the RNeasy Mini kit from Qiagen (Hilden, Germany). RNA integrity was confirmed by agarose gel electrophoresis. Total RNA $(1 \mu \mathrm{g})$ was reverse transcribed using oligo-dT primers $(0.5 \mu \mathrm{g})$ and Superscript II Reverse Transcriptase (Invitrogen), in a final volume of $20 \mu \mathrm{l}$ containing $1 \mathrm{mM}$ dNTP, $10 \mathrm{mM}$ DTT and first-strand buffer $(50 \mathrm{mM}$ Tris- $\mathrm{HCl}, \mathrm{pH} 8.3,75 \mathrm{mM} \mathrm{KCl}$, $3 \mathrm{mM} \mathrm{MgCl}_{2}$ ). After $50 \mathrm{~min}$ of incubation at $42^{\circ} \mathrm{C}$, the reactions were inactivated for $15 \mathrm{~min}$ at $70^{\circ} \mathrm{C}$. The cDNAs were purified with the High Pure PCR Product Purification kit (Roche Diagnostics) according to the manufacturer's instructions. In each cDNA sample, we measured the expression of gal-3 as well as that of two housekeeping genes, the transcription factor SP1 and TATA box-binding protein (TBP). The analysis of gal-3, SP1 and TBP expression in the different cDNA samples was performed by quantitative realtime PCR with the following primers: gal-3 sense, $5^{\prime}$-CAA TACAAAGCTGGATAATAACTGG- $3^{\prime}$ and gal-3 antisense, 5'-GATTGTACTGCAACAAGTGAG-3' (amplicon of $149 \mathrm{bp}$ ); SP1 sense, $5^{\prime}$-CCGCTCCCAACTTACAGAAC- $3^{\prime}$ and SP1 antisense, $5^{\prime}$-ATGATGTTGCCTCCACTTCC-3' (amplicon of $223 \mathrm{bp}$ ); TBP sense, $5^{\prime}$-TTCGGAGAGTTCTGGGATTG- $3^{\prime}$ and TBP antisense, $5^{\prime}$-AATCAGTGCCGTGGTTCGT- $3^{\prime}$ (amplicon of $175 \mathrm{bp}$ ). We used $20 \mathrm{ng}$ of cDNAs of each sample for analysis. Briefly, in a reaction, $2 \mu \mathrm{l}$ of cDNA (20 ng) were mixed with the primers (each at $0.5 \mu \mathrm{M}$ ) and the Lightcycler FastStart DNA Master SYBRGreen I reagents (Roche Diagnostics) in a $20 \mu$ l final volume. Reactions were carried out in a Lightcycler thermocycler instrument (Roche Diagnostics). In addition to the samples, each run included a standard curve for the gene analyzed. These standard curves consisted of PCRs performed on serial dilutions of a purified DNA fragment as substrate. All primers were from Eurogentec (Seraing, Belgium). Gal-3 mRNA expression was normalized by the geometric mean of the quantifications obtained for the housekeeping genes. The results are expressed as percentage of the control $(0 \mathrm{~h})$. 


\section{Generation of Stable Cell Lines Knockdown for Gal-3} The pSUPER and pSUPER-shGal3-551 vectors were a generous gift from Silvia Soddu (Regina Elena Cancer Institute, Rome, Italy). The pSUPER-shGal3-551 vector was previously shown to downregulate gal-3 expression. ${ }^{27}$ It contains the following sequence (conserved motif, underlined): GATCCC CCAACAGGAGAGTCATTGTTTTCAAGAGAAACAATGAC TCTCCTGTTGTTTTTGGAAA-3' (Gal3-551, sense) and 5'-AGCTTTTCCAAAAACAACAGGAGAGTCATTGTTTCTCT TGAAAACAATGACTCTCCTGTTGGGG-3' (Gal3-551, antisense). To create pSUPER-shScr, the following oligonucleotides were annealed and cloned into pSUPER (between the BglII and XhoI sites): 5'-GATCCCCGTTCAATTCAACGTA GATGTTCAAGAGACATCTACGTTGAATTGAACTTTTTGG $\overline{\text {AAC}^{\prime}} 3^{\prime}$ and $5^{\prime}$-TCGAGTTCCAAAAAGTTCAATTCAACG TAGATGTCTCTTGAACATCTACGTTGAATTGAACGGG-3' These oligonucleotides contain a sequence (underlined) that is a randomization of base pair 943-961 of the LGALS3 (gal-3) mRNA. The pSUPER-shScr plasmid was sequenced to confirm correct insertion of the oligonucleotides. The empty pSUPER as well as the pSUPER-shScr and pSUPER-shGal3551 vectors were co-transfected (at 10:1 ratio) with the pPUR vector ( $\mathrm{BD}$ Biosciences Clontech) that carries a selectable puromycin-resistance marker. ${ }^{28}$ The Fugene 6 transfection reagent (Roche Diagnostics) was used. Selection started at $48 \mathrm{~h}$ after transfections under $2 \mu \mathrm{g} / \mathrm{ml}$ puromycin. Independent clones were expanded and screened for gal-3 protein and mRNA expression. Out of 40 clones transfected with the pSUPER-shGal3-551 vector, 3 displayed a marked decrease of gal-3 mRNA expression (above 80\%), 2 of which, named shGal3-Cl1 and -Cl2, were subsequently used in our studies. Stable puromycin-resistant derivatives transfected with the empty pSUPER and the pSUPER-shScr vector were also cloned and had unchanged gal-3 protein level.

\section{Statistical Analysis}

The Mann-Whitney and the Kruskal-Wallis tests were used to compare independent groups of numerical data (for two groups and more than two groups, respectively). When the Kruskal-Wallis test was significant, post hoc tests (Dunn procedure) were used to compare the group pairs of interest in avoiding multiple comparison effects.

\section{RESULTS}

\section{The Conditioned Medium of LPS-Activated} Macrophages Contains High Level of M1 Type Cytokines To analyze the role of infiltrating macrophages on the development of colon cancer, we used an in vitro model in which monocytic THP-1 cells previously differentiated into macrophages with PMA are activated by a $24 \mathrm{~h}$ treatment with LPS. Table 1 indicates that CM-LAM contains elevated amounts of GM-CSF, IL-6, IL- 8 , TNF- $\alpha$ and IL- $\beta$ as compared to CM-UM. This is in agreement with data reported by others showing that LPS causes the M1 polarization of macrophages and the release of proinflammatory cytokines. $^{\text {13,14 }}$

\section{The Conditioned Medium of LPS-Activated Macrophages Exerts Growth-Inhibitory and Death-Inducing Activities on Colon Adenocarcinoma DLD-1 Cells}

The effect of CM-LAM on cell growth and viability of DLD-1 cells was first analyzed by video cellular microscopy (Figure 1). The recorded films highlight the marked morphological changes as well as the growth decrease occurring in the presence of $20 \%$ CM-LAM, as compared to untreated controls or cells cultivated in the presence of 20\% CM-UM. Such changes are already observed after $12 \mathrm{~h}$ of treatment (Figure 1a). Although DLD-1 control cells typically grow in small colonies or clusters, cells cultivated in the presence of 20\% CM-LAM detach from each other and only remain connected through multiple spindle-like membrane protrusions. At longer time points, such spindle connections disappear and the isolated cells became round, suggesting apoptotic cell death (Figure 1a). Based on the films images corresponding to time points $0,12,24,36$ and $48 \mathrm{~h}$, we evaluated the effects of the different treatments on cell growth by counting the number of cells. The number of cells increases linearly in the control condition. By comparison, the growth rate of DLD-1 cells treated with $20 \%$ CM-UM significantly decreases $(P=0.003$; Figure $1 \mathrm{~b})$. Nonetheless, treatment of DLD-1 cells with $20 \%$ CM-LAM not only drastically inhibits cell growth but strongly impairs cell survival. As compared to time $0 \mathrm{~h}$, the addition of CM-LAM led to a dramatic decrease in the number of cells that reaches $95 \%$ at $48 \mathrm{~h}$ of treatment $(P<0.001$; Figure $1 \mathrm{~b})$.

\section{The Conditioned Medium of LPS-Activated Macrophages Causes Apoptotic Cell Death}

To determine whether cell death occurred by apoptosis, we used the well-described Ann V binding assay coupled to a flow cytometry analysis which allows the distinction to be made between early (Ann V-positive/PI-negative cells) and late (Ann V-positive/PI-positive cells) apoptosis. Only a

Table 1 Validation of M1 response in LPS-activated macrophages

\begin{tabular}{lcc}
\hline & Unstimulated & LPS-activated \\
\hline GM-CSF & ND & 0.3 \\
IL-1 $\beta$ & 0.008 & 7.1 \\
IL-6 & 0.01 & 43.4 \\
IL-8 & 1.03 & 365.6 \\
TNF- $\alpha$ & ND & 167.5 \\
\hline
\end{tabular}

Monocytic THP-1 cells previously differentiated into macrophages by treatment with PMA for $48 \mathrm{~h}$ were activated with $1 \mu \mathrm{g} / \mathrm{ml}$ LPS. At $24 \mathrm{~h}$, the conditioned media were assayed for the presence of GM-CSF, IL-1 $\beta$, IL-6, IL-8 and TNF- $\alpha$ (ND: not determined). Concentrations are in $\mathrm{ng} / \mathrm{ml}$. 
a
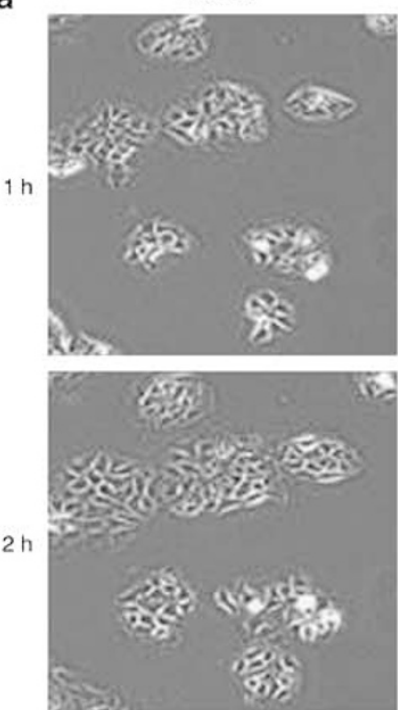

$12 \mathrm{~h}$

$24 \mathrm{~h}$
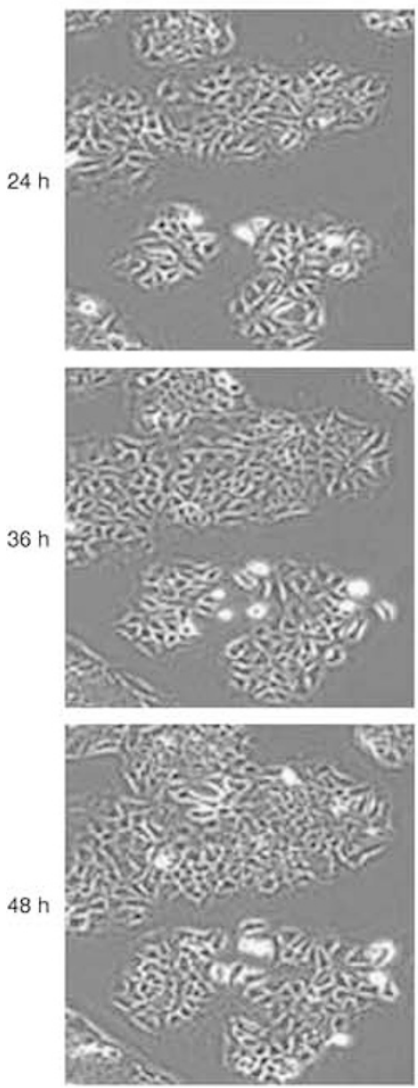

b 500
CM-UM
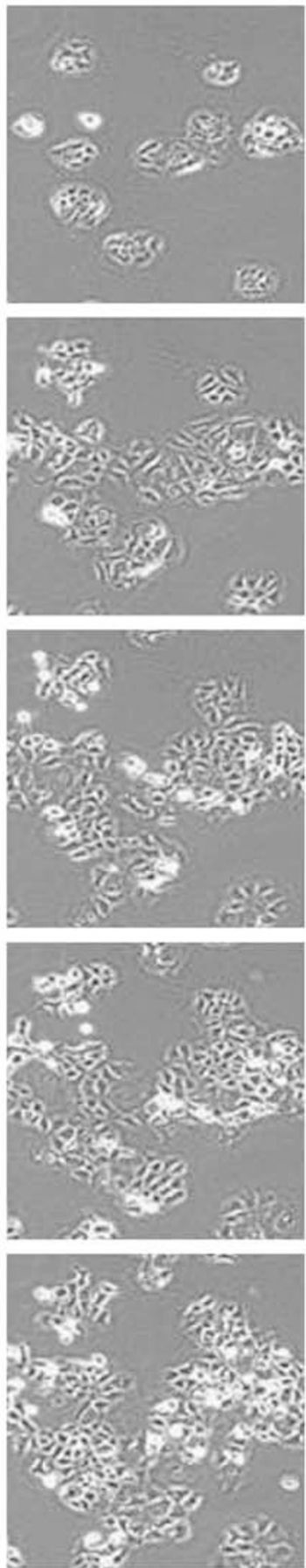

CM-LAM
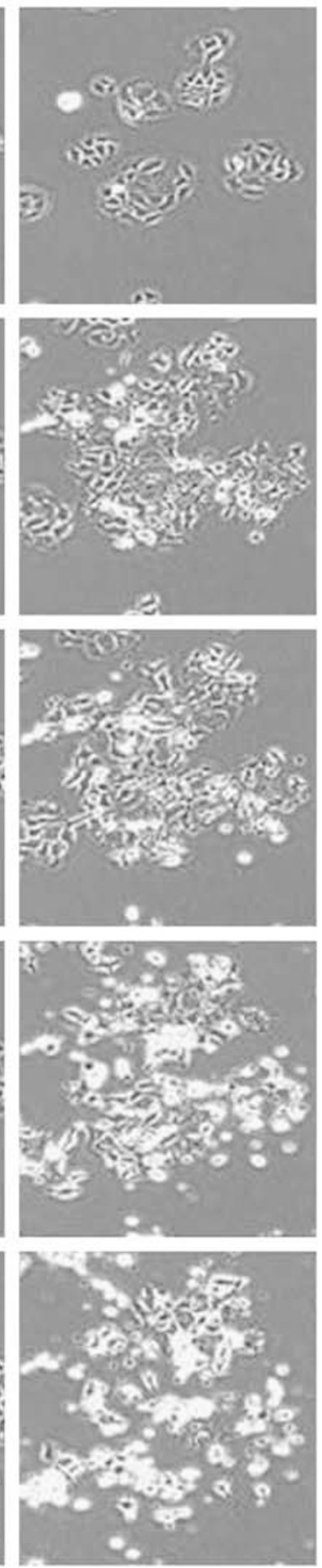

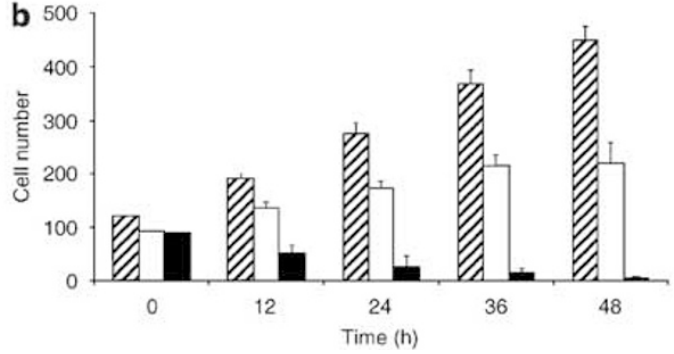


slight increase in the number of apoptotic cells was noted in the presence of $20 \%(\mathrm{v} / \mathrm{v}) \mathrm{CM}-\mathrm{UM}$ (Figure 2a). In contrast, marked apoptosis was observed when DLD-1 cells were incubated for $48 \mathrm{~h}$ in the presence of 20\% CM-LAM. By comparison to cells cultivated in $0.2 \%$ FBS medium, the percentage of early apoptotic cells increases by 11 -fold in cultures exposed to 20\% CM-LAM ( $P=0.003$; Figure 2a). Apoptotic cell death was confirmed by analyzing the caspasedependent cleavage of PARP. The cleaved form (p85-PARP) was clearly detectable after a $24 \mathrm{~h}$ incubation in the presence of $20 \%$ CM-LAM and its abundance further increases at later time points (48 and $72 \mathrm{~h}$ ), indicating apoptosis (Figure $2 \mathrm{~b}$ ).

\section{Decreased Expression of Gal-3 in DLD-1 Cells Treated with the Conditioned Medium of LPS-Activated Macrophages}

As described in the introduction, several articles report an association between gal-3 expression and the malignant properties of different kinds of cancer, including colon cancer, ${ }^{16-22}$ and this could be at least in part due to the antiapoptotic activities of this protein. ${ }^{29}$ As DLD-1 cells express a high level of gal-3, we investigated, at both the protein and mRNA level, whether the expression of gal-3 was modified following treatment with CM-LAM. Short treatments of 3 or $7 \mathrm{~h}$ had no effect on gal-3 mRNA expression. However, at $24 \mathrm{~h}$, a marked decrease of gal- 3 mRNA was observed in cells treated with $20 \%$ CM-LAM, as compared to the time point $0 \mathrm{~h}(P=0.004)$ or to cells incubated with CM-UM $(P=0.002$; Figure $3 \mathrm{a})$. This difference persists at 48 and $72 \mathrm{~h}$ (Figure $3 \mathrm{a}$ ). To analyze whether the decrease of gal-3 mRNA was associated with a decrease at the protein level, total protein lyzates were prepared from DLD-1 cells treated with $20 \%$ CM-UM or CM-LAM. No change in gal-3 expression was observed following treatment with CM-UM. In contrast, the expression of gal-3 was decreased after $24 \mathrm{~h}$ of incubation with $20 \%$ CM-LAM as well as at later time points (48 and $72 \mathrm{~h}$; Figure $3 \mathrm{~b}$ ).

\section{Determination of $\mathrm{IC}_{50}$ of the Conditioned Medium from LPS-Activated Macrophages}

$\mathrm{IC}_{50}$ (concentration inhibiting the overall growth by $50 \%$ ) was determined by treating DLD-1 cells for $72 \mathrm{~h}$ with various concentrations $(\mathrm{v} / \mathrm{v})$ of CM-UM or CM-LAM in $0.2 \%$ or in $10 \%$ FBS culture medium (Figure 4 ). We found that on DLD-1 cells, CM-LAM has an $\mathrm{IC}_{50}$ of $1.6 \%$ in $0.2 \%$ FBS culture medium (Figure 4a). It should also be pointed out that, as shown in Figure 1b, CM-UM displays a growth inhibitory activity, although minimal as compared to CM-LAM
(Figure 4a). In 10\% FBS culture medium, a higher dose of CM-LAM was required to achieve a similar growth inhibition. Indeed, in this culture condition, CM-LAM has an $\mathrm{IC}_{50}$ of $20 \%$ (Figure $4 \mathrm{~b}$ ).

\section{The Conditioned Medium of LPS-Activated Macrophages Modulates the Localization of Gal-3}

Gal-3 exerts different effects on cell survival and proliferation depending on its subcellular localization. For instance, its antiapoptotic activities are commonly linked to its presence in the cytoplasm..$^{29,30}$ In addition, extracellular gal-3 in the tumor microenvironment can markedly influence the tumorassociated immune response because the protein is proinflammatory. ${ }^{23,24}$ Using the $\mathrm{IC}_{50}$ determined above $(20 \% \mathrm{CM}$ LAM in $10 \%$ FBS culture medium, Figure $4 \mathrm{~b}$ ), we tested whether the secretion or subcellular localization of gal-3 was modified in response to incubation with CM-LAM. We first verified that CM-LAM, at the $\mathrm{IC}_{50}$ concentration, causes a decrease in gal-3 expression. Following incubation with $20 \%$ CM-LAM, gal-3 mRNA expression was downregulated at
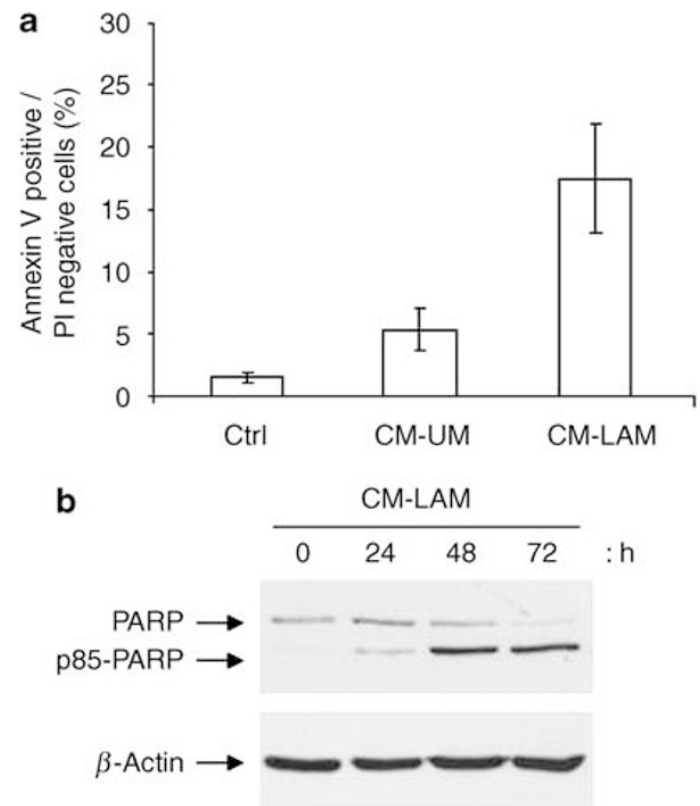

Figure 2 CM-LAM induces apoptosis. (a) Flow cytometry analysis of annexin $\mathrm{V}$ reactive cells. DLD-1 cells were incubated for $48 \mathrm{~h}$ in culture medium containing $0.2 \%$ FBS and the same medium supplemented with $20 \%(\mathrm{v} / \mathrm{v}$ ) of either CM-UM or CM-LAM. The graph shows the percentages of early apoptosis (cells positive for annexin $\mathrm{V}$ and negative for $\mathrm{PI}$ ). Data are mean of quadruplicates \pm s.d. (b) Western blot analysis of the cleavage of PARP in DLD-1 cells exposed for $0,24,48$ and $72 \mathrm{~h}$ to $20 \%$ CM-LAM in $0.2 \%$ FBS culture medium.

Figure 1 Effect of CM-LAM on DLD-1 cell growth and viability, using video cellular microscopy. (a) Recorded still images obtained by video cellular microscopy of time points 1, 12, 24, 36 and $48 \mathrm{~h}$. Cells were cultured for $48 \mathrm{~h}$ in $0.2 \%$ FBS culture medium (control) and the same medium supplemented

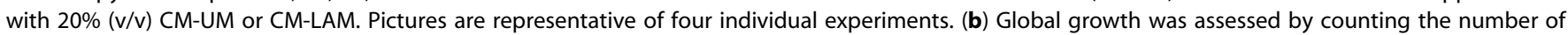
morphologically viable cells in the different conditions: control cells (dashed columns), cells incubated with CM-UM (white columns), cells treated with CM-LAM (black columns). Countings were based on the still images corresponding to time points $0,12,24,36$ and $48 \mathrm{~h}$. Data are representative of four individual experiments (mean \pm s.d.). 

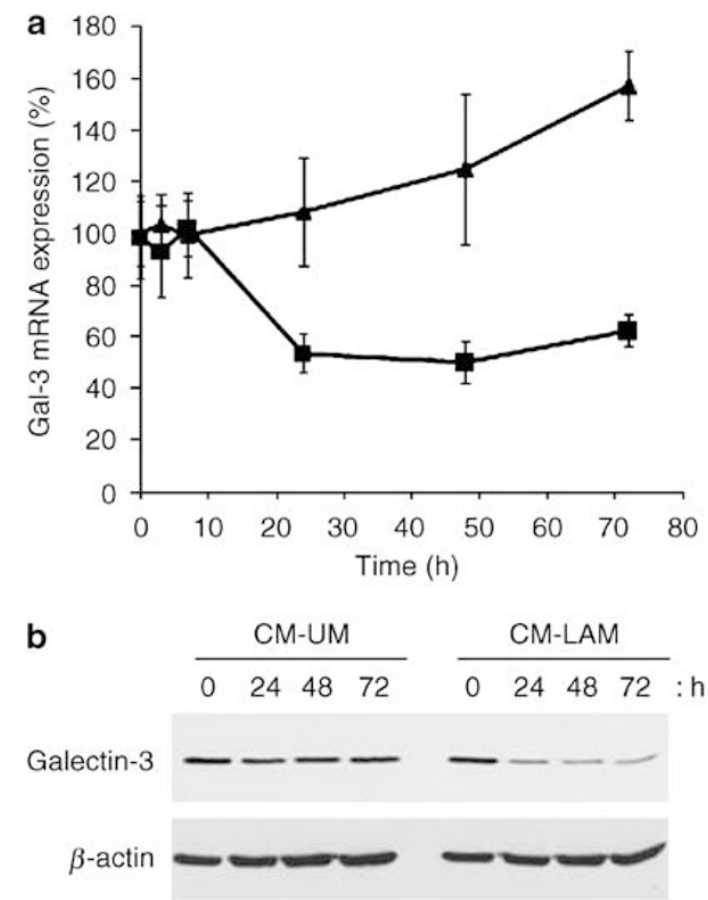

Figure 3 Decreased expression of gal-3 mRNA and protein after treatment with CM-LAM. (a) Effect of CM-LAM on gal-3 mRNA expression measured by real-time quantitative PCR. DLD-1 cells were incubated for $0,3,7,24,48$ and $72 \mathrm{~h}$ in $0.2 \% \mathrm{FBS}$ culture medium supplemented with $20 \%$ ( $/ \mathrm{v}$ ) CM-UM (triangles) or CM-LAM (squares). Gal-3 mRNA level is expressed as percentage of the control $(0 \mathrm{~h})$. Data are mean of sextuplicates $\pm \mathrm{s}$.d. (b) Western blot showing the gal-3 protein expression in DLD-1 cells incubated for $0,24,48$ and $72 \mathrm{~h}$ in $0.2 \%$ FBS culture medium supplemented with $20 \%(\mathrm{v} / \mathrm{v})$ of either CM-UM or CM-LAM. Representative data of three experiments are shown.

$24 \mathrm{~h}$ or later time points $(P=0.003$; Figure $5 \mathrm{a})$. Similarly, gal-3 protein level was decreased following a $72 \mathrm{~h}$ incubation in the presence of CM-LAM (Figure 5a). To assess gal-3 subcellular localization, we performed a cell fractionation in cells treated or not treated for $72 \mathrm{~h}$ with CM-LAM. The level of gal-3 was determined in both the nuclear and cytoplasmic fraction. The integrity of the fractions was monitored by performing a western blot for lamin $\mathrm{A} / \mathrm{C}$, a nuclear protein (Figure 5b). As compared to untreated control cells, a sharp decrease in nuclear gal-3 was observed. A decrease in cytoplasmic gal-3 was also noted, although modest as compared to that in the nuclear fraction (Figure 5b). These data can suggest either a differential degradation rate between the two compartments or an imbalance in the nuclear/cytoplasmic shuttling of gal-3. The gal-3 extracellular level was assessed by an ELISA. A higher secretion of extracellular gal-3 was observed in DLD-1 cells treated with 20\% CM-LAM as compared to cells incubated in the presence of $20 \%$ CM-UM (Figure $5 \mathrm{c}$ ). The concentration of gal-3 secreted by the DLD-1 cells was obtained by subtracting the gal-3 level recorded at $0 \mathrm{~h}$ (gal-3 secreted by the macrophages and already present in $20 \%$ CM-LAM and CM-UM prior the incubation) from the
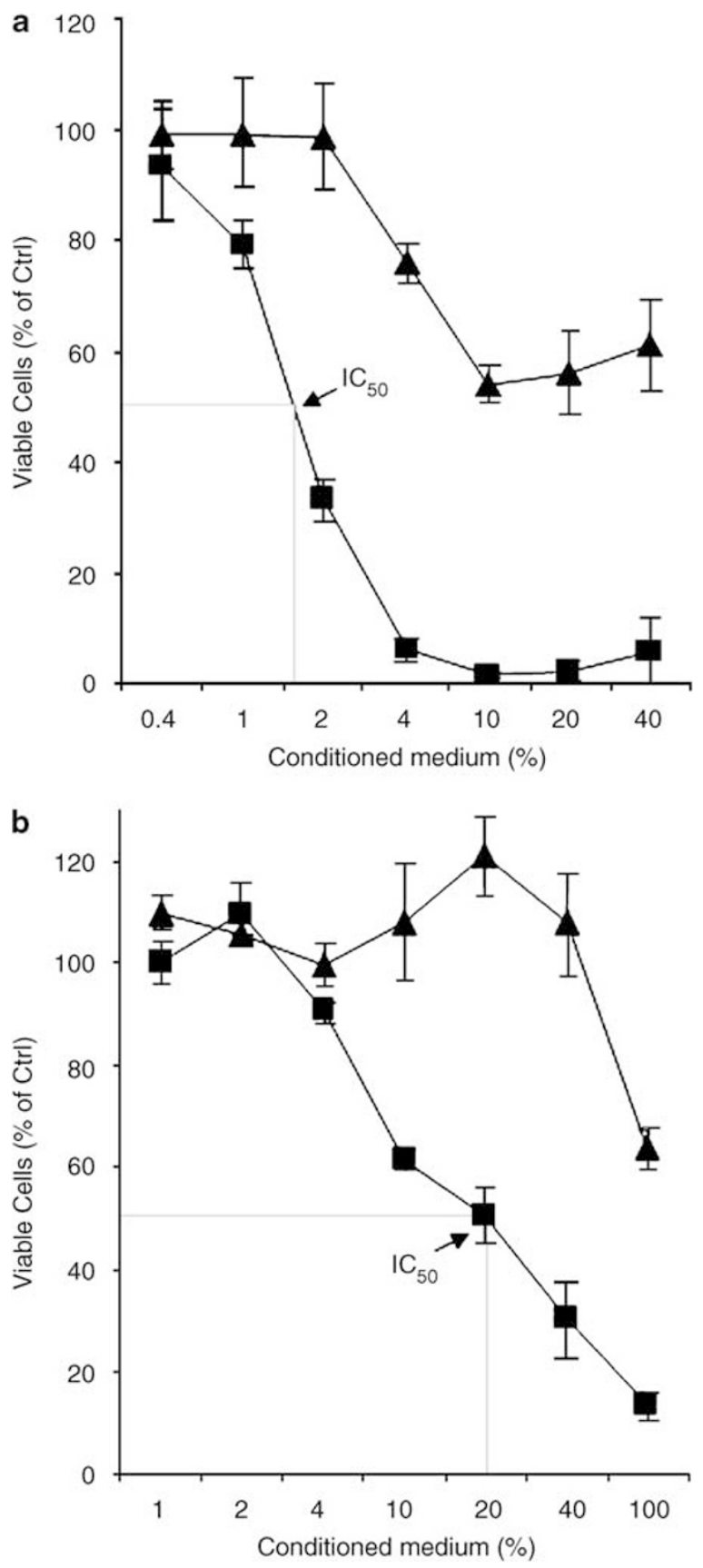

Figure $4 \mathrm{IC}_{50}$ determination of CM-LAM. DLD-1 cells were incubated for $72 \mathrm{~h}$ in culture medium containing different concentrations of CM-UM (triangles) or CM-LAM (squares). Incubations were performed in (a) $0.2 \%$ FBS culture medium and (b) 10\% FBS culture medium. Viability was assessed by MTT assay. Results are expressed as percentage of the control ( $0 \%$ conditioned media). Data are mean of quadruplicates \pm s.d.

gal-3 concentration at 48 or $72 \mathrm{~h}$. As shown in Figure 5c, CMLAM significantly increases the release of gal-3 by 2.4 -fold at $48 \mathrm{~h}(P=0.008)$ and 3.1 -fold at $72 \mathrm{~h}(P=0.008)$, as compared to CM-UM (Figure $5 \mathrm{c}$ ). We next tested whether extracellular gal-3 modulates the sensitivity to CM-LAM. DLD-1 cells were incubated for $72 \mathrm{~h}$ with $1.6 \%$ CM-LAM 
a
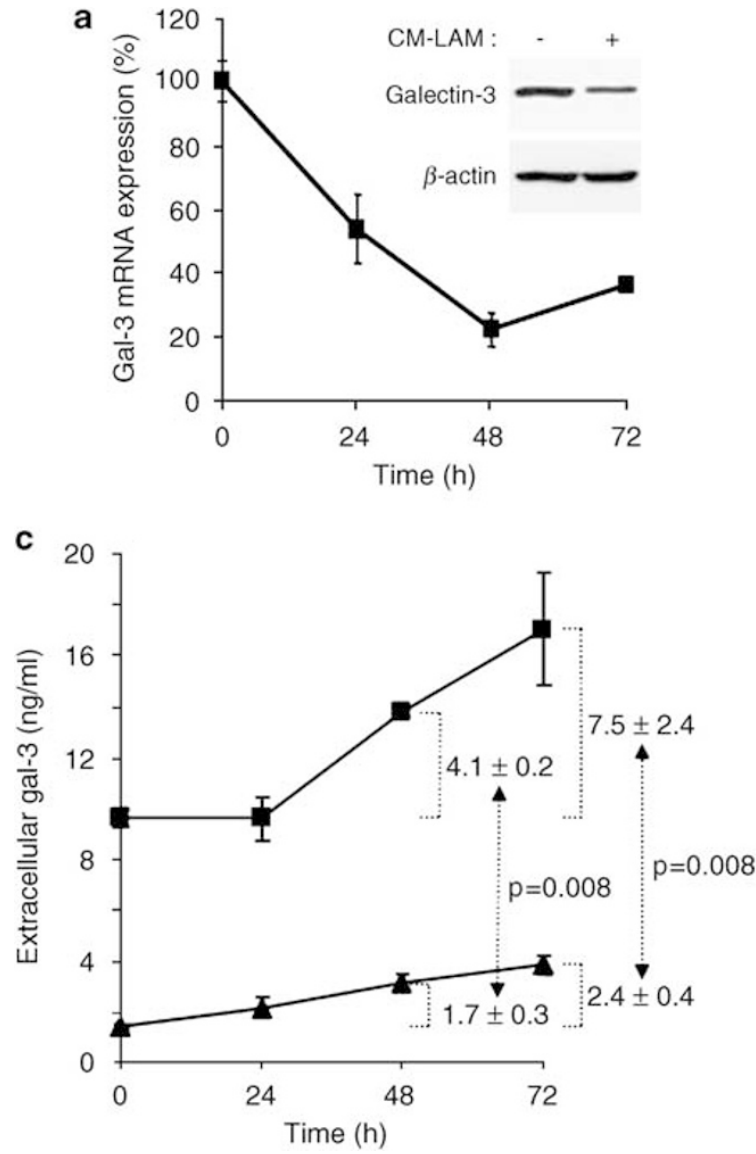

b

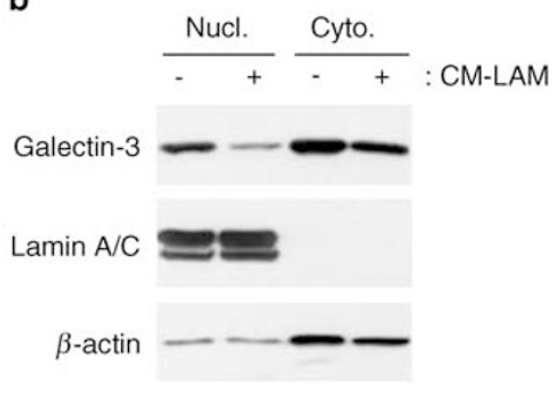

d

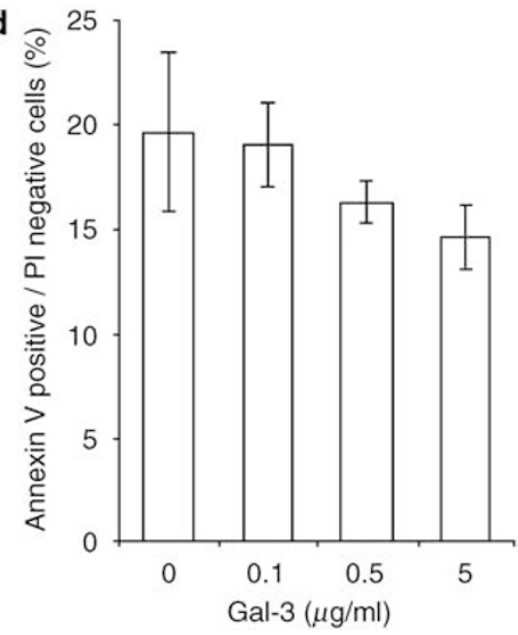

Figure 5 Analysis of the intracellular localization and the secretion of gal-3 in DLD-1 cells treated with CM-LAM. (a) Expression of gal-3 mRNA and protein following exposure of DLD-1 cells to $20 \% \mathrm{v} / \mathrm{v}$ CM-LAM in 10\% FBS culture medium. Gal-3 mRNA level was measured by real-time quantitative PCR and is expressed as percentage of the control $(0 \mathrm{~h})$. Data are mean of quadruplicates \pm s.d. Gal-3 protein level was assessed after a $72 \mathrm{~h}$ incubation in $10 \%$ FBS culture medium or the same medium supplemented with 20\% CM-LAM. (b) Effect of CM-LAM on the intracellular localization of gal-3. A cell fractionation was performed, as described in the 'Materials and Methods', in DLD-1 cells exposed for $72 \mathrm{~h}$ to $10 \%$ FBS culture medium or the same medium supplemented with $20 \%$ CM-LAM. Lamin A/C, a nuclear protein, is used as control for the integrity of the fractions. $\beta$-Actin is localized in both the nucleus and the cytoplasm. Data shown are representative of three experiments. (c) Secretion of gal-3 by DLD-1 cells in the presence of CM-LAM and CM-UM. Squares represent $20 \% \mathrm{v} / \mathrm{v}$ CM-LAM in $10 \%$ FBS culture medium; triangles, $20 \% \mathrm{v} / \mathrm{v}$ CM-UM in $10 \%$ FBS culture medium. The gal-3 concentration at $0 \mathrm{~h}$ is the gal-3 level present in the diluted conditioned media just prior the beginning of the incubation. Concentrations are in $\mathrm{ng} / \mathrm{ml}$. Data are mean of quintuplicates \pm s.d. (d) Effect of the extracellular gal-3 level on the sensitivity to CM-LAM. DLD-1 cells were incubated in $0.2 \%$ FBS culture medium supplemented with $1.6 \%(\mathrm{v} / \mathrm{v})$ CM-LAM and different doses of human recombinant gal-3 $(0,0.1,0.5$ and $5 \mu \mathrm{g} / \mathrm{ml})$. After $72 \mathrm{~h}$ of treatment, cells were harvested, labeled with annexin V-FITC (Ann V) and propidium iodide (PI) and analyzed by flow cytometry. The graph shows the percentages of early apoptosis (cells positive for annexin $\mathrm{V}$ and negative for PI). Data are mean of triplicates \pm s.d.

in $0.2 \%$ FBS culture medium ( $\mathrm{IC}_{50}$ concentration described in Figure 4a) supplemented with increasing amounts $(0,0.1$, 0.5 and $5 \mu \mathrm{g} / \mathrm{ml}$ ) of human recombinant gal-3 (Figure $5 \mathrm{~d}$ ). A decrease in the percentage of apoptotic cells was observed in the presence of extracellular gal-3 at concentrations of 0.5 and $5 \mu \mathrm{g} / \mathrm{ml}$ (Figure $5 \mathrm{~d}$ ). It must be stressed that the protective effect is only observed at concentrations much higher than those actually released by DLD-1 cells (Figure 5c).

\section{The Knockdown of Gal-3 Sensitizes DLD-1 Cells to the Conditioned Medium of LPS-Activated Macrophages}

As CM-LAM induced a significant decrease of the intracellular gal-3 protein level, we examined how such a decrease can affect the sensitivity to the proinflammatory/ apoptotic stimulus. To directly test that, we interfered with gal-3 expression by stably transfecting DLD-1 cells with the pSUPER-shGal3-551 vector, as described in 'Materials and Methods'. At the end of the selection process, we obtained stable clones displaying a greater than $80 \%$ decrease of gal-3 mRNA expression (Figure 6a). Knockdown clones shGal3$\mathrm{Cl} 1$ and $-\mathrm{Cl} 2$ were characterized for gal-3 protein expression. As compared to clones transfected with the empty pSUPER or the pSUPER-shScr vector, a $95 \%$ decrease of gal-3 protein level was observed (Figure $6 \mathrm{~b}$ ). To test how gal-3 knockdown affects the sensitivity to CM-LAM, we performed MTT assays using two control clones (pSUPER-Cl1 and pSUPER-shScr$\mathrm{Cl} 1)$ and the two gal-3 knockdown clones. After a $72 \mathrm{~h}$ exposure to 5,10 or $20 \%$ CM-LAM (in 10\% FBS culture 


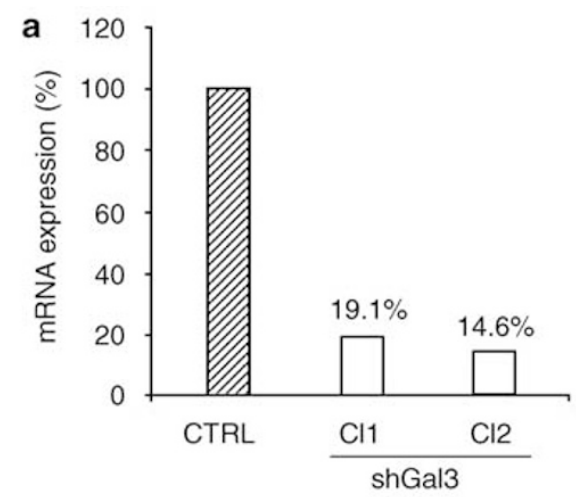

C

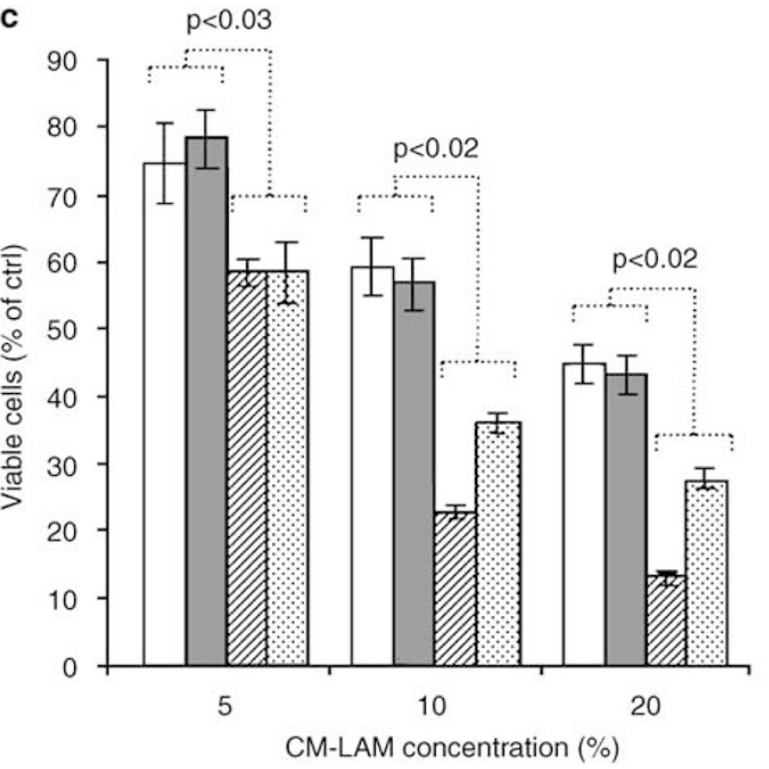

b

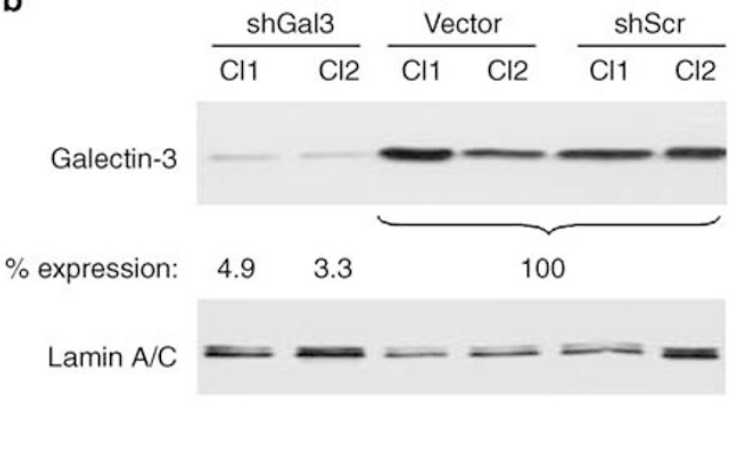

d

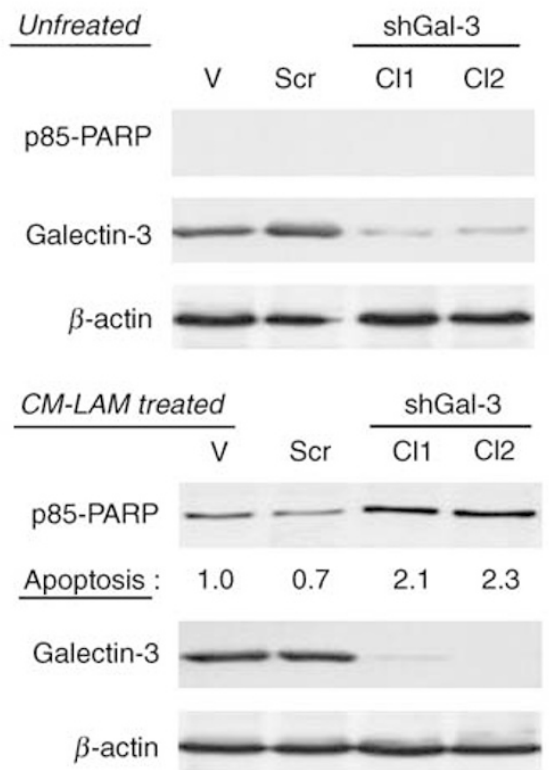

Figure 6 The knockdown of gal-3 sensitizes DLD-1 cells to CM-LAM. (a) Gal-3 mRNA expression of knockdown clones, shGal3-Cl1 and -Cl2. These clones were stably transfected with the vector pSUPER-shGal3-(551) as described in 'Materials and Methods'. Gal-3 mRNA level was measured by real-time quantitative PCR. The control (CTRL, 100\%) is the mean gal-3 mRNA expression of six control clones, three transfected with the empty vector and three transfected with pSUPER-shScr. Data are representative of two experiments. (b) Western blot of gal-3 protein level in clones shGal3-Cl1 and - $\mathrm{Cl} 2$, by comparison to clones transfected with the empty pSUPER vector (Vector) or the pSUPER-shScr vector (shScr). The residual gal-3 expression in the knockdown clones was assessed by a densitometry analysis of the gal-3 bands, and normalization to the loading control (lamin A/C). The value of $100 \%$ expression is given by the mean of the four control clones. (c) MTT assay showing the enhanced sensitivity to CM-LAM in gal-3 knockdown cells. Cells were incubated for $72 \mathrm{~h}$ in $10 \% \mathrm{FBS}$ culture medium containing $0,5,10$ or $20 \%$ CM-LAM. White columns indicate empty vector (CI1); gray columns, shScr vector (Cl1); hatched columns, shGal3-Cl1; dotted columns, shGal3-Cl2. Results are expressed as percentage of the control (0\% CM-LAM). Data are mean of sextuplicates \pm s.d. The displayed $P$-values result from the post hoc test and are the maximum $P$-values obtained for the different pairs involved in the brackets. (d) Western blot of the cleavage of PARP in untreated cells (top) or cells treated for $16 \mathrm{~h}$ with $20 \%$ CM-LAM in $10 \%$ FBS culture medium (bottom). The cleavage of PARP was analyzed in the gal-3 knockdown clones, shGal3-Cl1 and - $\mathrm{Cl} 2$, as well as two control clones- $\mathrm{V}$, empty vector (Cl1) and $\mathrm{Scr}$, shScr vector $(\mathrm{Cl} 1)$.

medium), we observed an increased sensitivity of the knockdown cells to CM-LAM, as compared to the control cells (Figure $6 \mathrm{c}$ ). The enhanced sensitivity was confirmed by a subsequent western blot analysis for the cleavage of PARP, a marker of apoptosis, after a $16 \mathrm{~h}$ exposure to $20 \%$ CM-LAM. In untreated cells, no PARP cleavage was detected (Figure 6d, top). Upon exposure to CM-LAM, PARP cleavage was clearly observed in the four clones. In addition, the cleavage of PARP was more abundant in cells knockdown for gal-3, as compared to control cells (Figure 6d, bottom). A densitometry analysis of the bands indicated a greater than twofold enhancement of the cleavage of PARP in the knockdown cells.

\section{DISCUSSION}

Macrophages constitute a significant part of the tumorinfiltrating immune cells and the increasing literature on the subject suggests that they can considerably affect the course of the disease. ${ }^{11,12}$ In most clinical studies, it has been observed that a high infiltration of the so-called TAMs correlate with a poor prognosis. ${ }^{11,12}$ High TAM density is often 
associated with a high vessel density, enhanced tumor invasiveness and metastasis occurrence, suggesting that they may behave as tumor promoters. ${ }^{11,12}$ Interestingly, in colon cancers, most articles suggest that TAMs prevent tumor development because patients with high TAMs have better prognosis and survival rate. ${ }^{7-9}$ This was evidenced recently by Forssell et $a l^{10}$ who performed a large study on 446 colorectal cancer specimens. Considering these findings, it is tempting to speculate that TAMs in colon cancer may not display a clear M2 polarization. As developed by Sica et al, ${ }^{12}$ the distinction between M1 and M2 macrophages does not encompass the entire spectrum of states that macrophages can express but rather represents two extremes. For instance, THP-1 monocytes that have been differentiated into macrophages with vitamin $\mathrm{D}_{3}$ are poor TNF- $\alpha$ producers but are able to generate substantial amount of superoxide anion, ${ }^{31}$ thus displaying both M1 and M2 characteristics.

Here we used an in vitro model to analyze the effects of culture supernatants from M1-polarized macrophages on colon cancer cells. In accordance with the literature, we found that activation of differentiated THP- 1 cells with LPS led to the release of proinflammatory cytokines such as IL- $1 \beta$ and TNF- $\alpha .{ }^{13,14}$ When applied to DLD- 1 colon adenocarcinoma cells, the culture supernatants obtained from these LPSactivated macrophages displayed a strong growth inhibitory activity and caused extensive cell death. Cell death occurred, at least partly, by apoptosis as evidenced by Ann V binding assays and PARP cleavage, in accordance with the high level of cytokines contained in CM-LAM known to be proapoptotic to susceptible tumor cells. ${ }^{32,33}$

DLD-1 colon adenocarcinoma cells express a high level of gal-3 in both their cytoplasms and nuclei. We found that CM-LAM induces a marked decrease of the expression of gal3 in DLD-1 cells at both the mRNA and protein levels. Interestingly, cell fractionation indicated that the decrease in gal-3 protein was more pronounced in the nucleus as compared to the cytoplasm, suggesting either an imbalance of the degradation rate between the two compartments or alternatively a modification in the nuclear/cytoplasmic shuttling of the protein. The effect of cytokines or inflammatory stimuli on gal-3 expression is a matter of controversy and likely depends on the experimental model used or the cell line studied. For instance, the expression of gal-3 is downregulated in the intestinal epithelia of Crohn's disease patients. ${ }^{34}$ Furthermore, TNF- $\alpha$ decreases the level of gal-3 mRNA in the human intestinal epithelial cell line HCT- $8 .{ }^{34}$ In contrast, proteome analysis of isolated Langerhans rat islets exposed to IL- $1 \beta$ identified gal- 3 as the most upregulated protein. ${ }^{35}$ Upregulation of gal-3 mRNA expression in human islets treated with IL- $1 \beta$, TNF- $\alpha$, IFN- $\gamma$ or a mix of these cytokines was also reported. ${ }^{35}$ We report that knocking down gal-3 in DLD- 1 cells results in a sensitization to CM-LAM, as evidenced by MTT assays and PARP cleavage. This is in accordance with different articles describing the antiapoptotic activity of gal-3, in response to a wide range of apoptotic insults such as staurosporine, anti-fas antibodies, nitric oxide, radiation, anoikis and TNF- $\alpha .{ }^{29,30}$

Last, we found that DLD-1 cells treated with CM-LAM secrete an increased amount of gal-3. Although, the protective effect of extracellular gal-3 during CM-LAM treatment was only observed at very high concentrations (more than 60 -fold higher than what is actually released by DLD- 1 cells), this observation remains interesting with regard to the involvement of gal-3 in the tumor-associated immune response. Gal-3 has been shown to be chemoattractive for monocytes and macrophages and is able to trigger an oxidative burst in macrophages. ${ }^{36}$ Nishi et al ${ }^{37}$ recently demonstrated that PMA-differentiated THP- 1 cells incubated with recombinant gal-3 release TNF- $\alpha$ and IL-8 (but not IL-4, IL-13 or TGF- $\beta$ ) at levels similar to what LPS induces. It therefore appears that in vivo an amplification loop could take place in which activated macrophages would, through the release of proinflammatory cytokines, induce the secretion of gal-3 by colon cancer cells. This secreted gal-3 would in turn stimulate the release of different factors by macrophages as well as recruit more macrophages because it is chemoattractive for this type of cells.

To conclude, the role of TAMs in tumorigenesis is complex because their behavior is modulated by the tumor microenvironment itself. Various factors are involved in the crosstalks that exist between TAMs and cancer cells and this report emphasizes that gal-3 is involved in this process. Indeed activated macrophages are able to modulate both the expression and the secretion of gal- 3 by colon cancer cells. It will be crucial to determine the phenotype of TAMs in colon cancer, with regard to the M1 and M2 types of polarization, because this will allow the development of in vitro models that are closer to the reality of the disease and will help to better understand why high TAMs densities are associated with a good prognosis in colon cancer.

\section{ACKNOWLEDGEMENTS}

This work was supported by the Fonds Yvonne Boël (Brussels, Belgium). Christine Decaestecker is a Senior Research Associate with the FNRS (Fonds National pour la Recherche Scientifique). We thank Unibioscreen SA (Brussels, Belgium) for performing the video cellular microscopy. We also thank Benoit Martin for analyzing the video microscopy data and Blair Jenkins for English editing on this paper.

\section{DISCLOSURE/DUALITY OF INTEREST}

None.

1. Dunn GP, Old LU, Schreiber RD. The immunobiology of cancer immunosurveillance and immunoediting. Immunity 2004;21:137-148.

2. Smyth MJ, Dunn GP, Schreiber RD. Cancer immunosurveillance and immunoediting: the roles of immunity in suppressing tumor development and shaping tumor immunogenicity. Adv Immunol 2006;90:1-50.

3. Philip $\mathrm{M}$, Rowley $\mathrm{DA}$, Schreiber $\mathrm{H}$. Inflammation as a tumor promoter in cancer induction. Semin Cancer Biol 2004;14:433-439.

4. de Visser KE, Korets LV, Coussens LM. De novo carcinogenesis promoted by chronic inflammation is B lymphocyte dependent. Cancer Cell 2005;7:411-423. 
5. Balkwill F, Mantovani A. Inflammation and cancer: back to Virchow? Lancet 2001;357:539-545.

6. Coussens LM, Werb Z. Inflammation and cancer. Nature 2002;420: 860-867.

7. Nakayama $\mathrm{Y}$, Nagashima N, Minagawa N, et al. Relationships between tumor-associated macrophages and clinicopathological factors in patients with colorectal cancer. Anticancer Res 2002;22:4291-4296.

8. Tan SY, Fan Y, Luo HS, et al. Prognostic significance of cell infiltrations of immunosurveillance in colorectal cancer. World J Gastroenterol 2005;11:1210-1214.

9. Funada $Y$, Noguchi $T$, Kikuchi R, et al. Prognostic significance of CD8+ T cell and macrophage peritumoral infiltration in colorectal cancer. Oncol Rep 2003;10:309-313.

10. Forssell J, Oberg A, Henriksson ML, et al. High macrophage infiltration along the tumor front correlates with improved survival in colon cancer. Clin Cancer Res 2007;13:1472-1479.

11. Lewis CE, Pollard JW. Distinct role of macrophages in different tumor microenvironments. Cancer Res 2006:66:605-612.

12. Sica A, Schioppa T, Mantovani A, et al. Tumour-associated macrophages are a distinct M2 polarised population promoting tumour progression: potential targets of anti-cancer therapy. Eur J Cancer 2006;42:717-727.

13. Abe T, Ohno M, Sato $T$, et al. 'Differentiation induction' culture of human leukemic myeloid cells stimulates high production of macrophage differentiation inducing factor.. Cytotechnology 1991;5:S75-S93.

14. Simbirtsev A, Prokopieva E, Ivanova E, et al. Interleukin-1 and tumour necrosis factor production by human monocytoid cells: study on a single cell level. Eur Cytokine Netw 1992;3:421-428.

15. Auwerx J. The human leukemia cell line, THP-1: a multifacetted mode for the study of monocyte-macrophage differentiation. Experientia 1991;47:22-31.

16. Legendre $\mathrm{H}$, Decaestecker $\mathrm{C}$, Nagy $\mathrm{N}$, et al. Prognostic values of galectin-3 and the macrophage migration inhibitory factor (MIF) in human colorectal cancers. Mod Pathol 2003;16:491-504.

17. Nagy $\mathrm{N}$, Legendre $\mathrm{H}$, Engels $\mathrm{O}$, et al. Refined prognostic evaluation in colon carcinoma using immunohistochemical galectin fingerprinting. Cancer 2003;97:1849-1858.

18. Hittelet $A$, Legendre $H$, Nagy $N$, et al. Upregulation of galectins-1 and 3 in human colon cancer and their role in regulating cell migration. Int J Cancer 2003;103:370-379.

19. Nakamura $\mathrm{M}$, Inufusa $\mathrm{H}$, Adachi $\mathrm{T}$, et al. Involvement of galectin-3 expression in colorectal cancer progression and metastasis. Int J Onco 1999;15:143-148.

20. Endo K, Kohnoe S, Tsujita E, et al. Galectin-3 expression is a potent prognostic marker in colorectal cancer. Anticancer Res 2005;25: 3117-3121.

21. Dumic J, Dabelic S, Flogel M. Galectin-3: an open-ended story. Biochim Biophys Acta 2006;1760:616-635.
22. Califice S, Castronovo V, Van Den Brüle F. Galectin-3 and cancer (review). Int J Oncol 2004;25:983-992.

23. Rabinovich GA, Baum LG, Tinari $\mathrm{N}$, et al. Galectins and their ligands: amplifiers, silencers or tuners of the inflammatory response? Trends Immunol 2002;23:313-320.

24. Sano H, Hsu DK, Yu L, et al. Human galectin-3 is a novel chemoattractant for monocytes and macrophages. J Immunol 2000;165:2156-2164.

25. Perillo NL, Marcus ME, Baum LG. Galectins: versatile modulators of cell adhesion, cell proliferation, and cell death. J Mol Med 1998;76: 402-412.

26. Nagy N, Brenner C, Markadieu N, et al. S100A2, a putative tumor suppressor gene, regulates in vitro squamous cell carcinoma migration. Lab Invest 2001;81:599-612.

27. Cecchinelli B, Lavra L, Rinaldo C, et al. Repression of the antiapoptotic molecule galectin-3 by homeodomain-interacting protein kinase 2 -activated p53 is required for p53-induced apoptosis. Mol Cell Biol 2006;26:4746-4757.

28. Paik J, Duncan T, Lindahl T, et al. Sensitization of human carcinoma cells to alkylating agents by small interfering RNA suppression of 3-alkyladenine-DNA glycosylase. Cancer Res 2005;65: 10472-10477.

29. Nakahara S, Oka N, Raz A. On the role of galectin-3 in cancer apoptosis. Apoptosis 2005; 10:267-275.

30. Nangia-Makker $P$, Nakahara $S$, Hogan V, et al. Galectin-3 in apoptosis, a novel therapeutic target. J Bioenerg Biomembr 2007;39:79-84.

31. Schwende $H$, Fitzke $E$, Ambs $P$, et al. Differences in the state of differentiation of THP-1 cells induced by phorbol ester and 1,25dihydroxyvitamin D3. J Leukoc Biol 1996;59:555-561.

32. Vaculova A, Hofmanova J, Soucek K, et al. Tumor necrosis factor-alpha induces apoptosis associated with poly(ADP-ribose) polymerase cleavage in HT-29 colon cancer cells. Anticancer Res 2002;22: 1635-1639.

33. Castigli E, Arcuri C, Giovagnoli L, et al. Interleukin-1beta induces apoptosis in GL15 glioblastoma-derived human cell line. Am J Physiol Cell Physiol 2000;279:C2043-C2049.

34. Jensen-Jarolim E, Gscheidlinger R, Oberhuber G, et al. The constitutive expression of galectin-3 is downregulated in the intestinal epithelia of Crohn's disease patients, and tumour necrosis factor alpha decreases the level of galectin-3-specific mRNA in HCT-8 cells. Eur J Gastroenterol Hepatol 2002;14:145-152.

35. Karlsen AE, Størling ZM, Sparre T, et al. Immune-mediated beta-cell destruction in vitro and in vivo-A pivotal role for galectin-3. Biochem Biophys Res Commun 2006;344:406-415.

36. Sato $S$, Nieminen J. Seeing strangers or announcing 'danger': galectin-3 in two models of innate immunity. Glycoconj J 2004;19: 583-591.

37. Nishi $\mathrm{Y}$, Sano $\mathrm{H}$, Kawashima $\mathrm{T}$, et al. Role of galectin-3 in human pulmonary fibrosis. Allergol Int 2007;56:57-65. 